\title{
Valoración de los resultados del Índice Sintético de la calidad educativa (ISCE) 2017 de las instituciones oficiales en el municipio de Soacha- Cundinamarca
}

\section{Evaluation of the results of the Synthetic Index of educational quality (ISCE) 2017 of the official institutions in the municipality of Soacha-Cundinamarca}

Jorge Enrique Díaz Pinzón

Universidad Nacional de Colombia, Colombia

Autor para correspondencia: jediazp@unal.edu.co,jorgediaz333@gmail.com

Fecha de recepción: 7 de Septiembre de 2017 - Fecha de aceptación: 15 de Noviembre de 2017

\section{Resumen}

Este trabajo de investigación tuvo por objetivo comparar si hay diferencias entre las instituciones educativas del municipio de Soacha-Cundinamarca y el resultado obtenido en el Índice Sintético de Calidad Educativa (ISCE) en al año 2017, además de comparar los promedios obtenidos por las diferentes instituciones educativas del municipio de Soacha-Cundinamarca. Se realizó una comparación múltiple entre medias independientes, a partir del análisis estadístico de los datos aplicando la prueba T-Student, se pudo observar que hay evidencia para rechazar la hipótesis alterna, por tanto las medias son diferentes. La institución educativa con menor promedio del ISCE fue Compartir con 4,5767 y la de mejor promedio fue la institución educativa Eugenio Díaz Castro con un promedio de 6,8667 .

Palabras clave: educación; calidad de la educación; evaluación comparativa; educación comparada

\begin{abstract}
This research aimed to compare if there are differences between the educational institutions of the municipality of Soacha-Cundinamarca and the results obtained in the Synthetic Quality of Education Index (ISCE) in 2017, in addition to comparing the averages obtained by the different institutions Educational institutions of the municipality of Soacha-Cundinamarca. We performed a multiple comparison between independent means, from the statistical analysis of the data using the T-Student test, it was observed that there is evidence to reject the alternative hypothesis, so the means are different. The educational institution with the lowest ISCE average was Share with 4.5767 and the highest average was the educational institution Eugenio Díaz Castro with an average of 6.8667 .
\end{abstract}

Key words: education; quality of education; comparative evaluation; comparative education 


\section{Introducción}

El 25 de Marzo de 2015, el Ministerio de Educación Nacional exhibió el Índice Sintético de Calidad Educativa (ISCE) como un instrumento diferente de medición de la calidad educativa en el país.(Zambrano, 2015). El Índice Sintético de Calidad Educativa-ISCE, nació como una medida sencilla de la calidad de la educación, con el propósito de los índices en educación es que los niños vayan a la escuela y que aprendan lo que tienen que aprender, esto con el fin de que Colombia sea el país más educado de América Latina en el 2025. (Godoy, 2015). Con ella, sabremos con confianza cómo somos en cada uno de nuestros ciclos educativos - Básica Primaria, Básica Secundaria y Media. (MEN, 2017)

A partir de los resultados del Índice Sintético de la Calidad Educativa (ISCE), los cuales disponen una herramienta fundamental para el establecimiento de metas y acciones de progreso, la determinación del impacto de las mismas y el involucramiento de la comunidad educativa en esta ruta hacia la excelencia. (Jaramillo, 2017). Antes que nada, es importante entender qué es el ISCE. Según el Ministerio de Educación es una herramienta que apoya en la búsqueda de la mejora de todos los colegios. (El Tiempo, 2017).

El Índice está determinado por una escala del 1 al 10, siendo 10 el valor más alto que se puede obtener. El puntaje tiene en cuenta cuatro componentes, y, al sumarlos, se obtiene el puntaje total del Índice Sintético de Calidad Educativa (ISCE). (Godoy, 2015)

Según el (MEN, 2017), el nuevo Índice Sintético de Calidad Educativa se llama "sintético" porque tiene cuatro componentes claves para mejorar son compuestos en uno solo. Estos son:

Progreso: Muestra qué tanto ha mejorado nuestra institución educativa en relación con el año anterior. Este componente da un máximo de 4 puntos de los 10 posibles que un establecimiento educativo puede obtener en el ISCE.

Desempeño: Refleja el puntaje promedio que los estudiantes obtuvieron en las Pruebas Saber 2016 para Matemáticas y Lenguaje, dando un máximo de 4 puntos de los 10 posibles que un establecimiento educativo puede obtener en el ISCE.

Eficiencia: Concierne a la proporción de alumnos que aprueban el año escolar y pasan al año siguiente. Para primaria y secundaria el valor de eficiencia corresponde al valor reportado por cada colegio en SIMAT sobre su tasa de aprobación (es decir dará máximo 1 punto en el ISCE).

Ambiente escolar: Concierne a la evaluación de las condiciones propicias para el aprendizaje en el aula de clase. Este componente que máximo dará 1 punto de los 10 posibles que un establecimiento educativo puede obtener en el ISCE.

\section{Población}

Esta investigación se centró en las 21 instituciones educativas oficiales del municipio de Soacha-Cundinamarca, con la información del ISCE para el año 2017. 


\section{Resultados}

\section{Hipótesis con el ANOVA}

La hipótesis del presente trabajo de investigación se diseña como una relación causal y se enuncia de la siguiente forma:

Hipótesis Alterna (Ha): Hay diferencias en el promedio de los puntajes obtenidos del Índice Sintético de Calidad Educativa entre las diferentes instituciones educativas oficiales del municipio de Soacha-Cundinamarca.

Hipótesis Nula (Ho): No hay diferencias en el promedio de los puntajes obtenidos del Índice Sintético de Calidad Educativa entre las diferentes instituciones educativas oficiales del municipio de Soacha-Cundinamarca.

4. Prueba Estadística: El valor de significancia de la prueba es de $\alpha=0.05$ (5\%), si es mayor se acepta la hipótesis nula si es menor se rechaza la hipótesis nula. Comparación entre medias independientes, se utilizó el Software SPSS v 20.0.

En la tabla 1, se observa que el p-valor de la prueba es igual a 0,12 es menor a $\alpha=0.05$, de esta manera se rechaza Ho, es decir que para al menos uno de los promedios es diferente de los demás. En la tabla 2 se aprecian las medias del Índice Sintético de la Calidad Educativa para las instituciones educativas oficiales del municipio de Soacha-Cundinamarca.

Tabla 1. ANOVA de un factor

\begin{tabular}{lrrrrr}
\hline Índice Sintético de Calidad Educativa & & & & & \\
& Suma de cuadrados & gl & Media cuadrática & F & Sig. \\
\hline Inter-grupos & 49,712 & 20 & 2,486 & 2,278 &, 012 \\
Intra-grupos & 45,833 & 42 & 1,091 & & \\
Total & 95,545 & 62 & & & \\
\hline
\end{tabular}

Tabla 2. Media del Índice Sintético de Calidad Educativa

\begin{tabular}{ccccc}
\hline Institución Educativa & $\mathrm{N}$ & Media & Mínimo & Máximo \\
& & & & \\
\hline Ciudad Latina & 3 & 4,5767 & 4,31 & 4,91 \\
Nuevo Compartir & 3 & 4,2367 & 4,20 & 4,27 \\
Compartir & 3 & 4,1900 & 3,94 & 4,32 \\
Santa Ana & 3 & 6,1667 & 5,13 & 7,49 \\
Integrado & 3 & 5,8700 & 4,40 & 7,37 \\
General Santander & 3 & 6,0933 & 4,70 & 7,42 \\
La Despensa & 3 & 4,7567 & 4,21 & 5,76 \\
Manuela Beltrán & 3 & 5,4000 & 4,85 & 6,11 \\
León XIII & 3 & 4,9100 & 4,16 & 6,13 \\
Buenos Aires & 3 & 4,2033 & 4,15 & 4,27 \\
Ciudadela Sucre & 3 & 4,4467 & 4,12 & 5,00 \\
Julio Cesar Turbay & 3 & 4,5533 & 4,11 & 4,99 \\
Luis Carlos Galán & 3 & 4,3200 & 4,08 & 4,70 \\
Gabriel García Márquez & 3 & 4,0133 & 3,83 & 4,24 \\
\hline
\end{tabular}




\begin{tabular}{ccccc}
\hline Cazucá & 3 & 6,0533 & 5,11 & 7,45 \\
El Bosque & 3 & 5,6233 & 4,35 & 7,38 \\
San Mateo & 3 & 7,0233 & 5,72 & 7,90 \\
Ricaurte & 3 & 5,2200 & 4,03 & 7,40 \\
Las Villas & 3 & 5,7667 & 4,53 & 7,31 \\
Eduardo Santos & 3 & 5,7200 & 4,44 & 7,37 \\
Eugenio Díaz Castro & 3 & 6,8667 & 5,59 & 7,56 \\
Total & 63 & 5,2386 & 3,83 & 7,90 \\
\hline
\end{tabular}

Ahora cuando Ho se rechaza en la prueba ANOVA, entonces se procede a realizar una prueba Post Hoc, en este caso la prueba de Diferencia Mínima Significativa (DMS) para muestras independientes y observar si hay diferencias entre cada uno de los niveles, como se puede observar en la tabla 3.

Tabla 3. Comparaciones múltiples. Diferencia Mínima Significativa

\begin{tabular}{|c|c|c|}
\hline (I) Colegio & (J) Colegio & Diferencia de medias (I-J) \\
\hline Ciudad & San Mateo & $-2,44667^{*}$ \\
\hline \multirow[t]{3}{*}{ Latina } & Eugenio Díaz Castro & $-2,29000^{*}$ \\
\hline & Santa Ana & $-1,93000^{*}$ \\
\hline & General Santander & $-1,85667^{*}$ \\
\hline Nuevo & Cazucá & $-1,81667^{*}$ \\
\hline \multirow[t]{2}{*}{ Compartir } & San Mateo & $-2,78667^{*}$ \\
\hline & Eugenio Díaz Castro & $-2,63000^{*}$ \\
\hline \multirow[t]{5}{*}{ Compartir } & Santa Ana & $-1,97667^{*}$ \\
\hline & General Santander & $-1,90333^{*}$ \\
\hline & Cazucá & $-1,86333^{*}$ \\
\hline & San Mateo & $-2,83333^{*}$ \\
\hline & Eugenio Díaz Castro & $-2,67667^{*}$ \\
\hline \multirow[t]{5}{*}{ Santa Ana } & Nuevo Compartir & $1,93000^{*}$ \\
\hline & Compartir & $1,97667^{*}$ \\
\hline & Buenos Aires & $1,96333^{*}$ \\
\hline & Luis Carlos Galán & $1,84667^{*}$ \\
\hline & Gabriel García M. & $2,15333^{*}$ \\
\hline \multirow[t]{3}{*}{ Integrado } & Gabriel García M. & $1,85667^{*}$ \\
\hline & Nuevo Compartir & $1,85667^{*}$ \\
\hline & Compartir & $1,90333^{*}$ \\
\hline General & Buenos Aires & $1,89000^{*}$ \\
\hline \multirow[t]{2}{*}{ Santander } & Luis Carlos Galán & $1,77333^{*}$ \\
\hline & Gabriel García M. & $2,08000^{*}$ \\
\hline La & San Mateo & $-2,26667^{*}$ \\
\hline \multirow[t]{2}{*}{ Despensa } & Eugenio Díaz Castro & $-2,11000^{*}$ \\
\hline & San Mateo & $-2,11333^{*}$ \\
\hline \multirow[t]{3}{*}{ León XIII } & Eugenio Díaz Castro & $-1,95667^{*}$ \\
\hline & Santa Ana & $-1,96333^{*}$ \\
\hline & General Santander & $-1,89000^{*}$ \\
\hline Buenos & Cazucá & $-1,85000^{*}$ \\
\hline \multirow[t]{2}{*}{ Aires } & San Mateo & $-2,82000^{*}$ \\
\hline & Eugenio Díaz Castro & $-2,66333^{*}$ \\
\hline Ciudadela & San Mateo & $-2,57667^{*}$ \\
\hline \multirow[t]{2}{*}{ Sucre } & Eugenio Díaz Castro & $-2,42000^{*}$ \\
\hline & San Mateo & $-2,47000^{*}$ \\
\hline \multirow[t]{2}{*}{ J.C. Turbay } & Eugenio Díaz Castro & $-2,31333^{*}$ \\
\hline & Santa Ana & $-1,84667^{*}$ \\
\hline
\end{tabular}




\begin{tabular}{|c|c|c|}
\hline Luis & General Santander & $-1,77333^{*}$ \\
\hline Carlos & Cazucá & $-1,73333^{*}$ \\
\hline \multirow[t]{3}{*}{ Galán } & San Mateo & $-2,70333^{*}$ \\
\hline & Eugenio Díaz Castro & $-2,54667^{*}$ \\
\hline & Santa Ana & $-2,15333^{*}$ \\
\hline Gabriel & Integrado & $-1,85667^{*}$ \\
\hline García & General Santander & $-2,08000^{*}$ \\
\hline \multirow[t]{5}{*}{ Márquez } & Cazucá & $-2,04000^{*}$ \\
\hline & San Mateo & $-3,01000^{*}$ \\
\hline & Las Villas & $-1,75333^{*}$ \\
\hline & Eugenio Díaz Castro & $-2,85333^{*}$ \\
\hline & Nuevo Compartir & $1,81667^{*}$ \\
\hline \multirow[t]{7}{*}{ Cazucá } & Compartir & $1,86333^{*}$ \\
\hline & Buenos Aires & $1,85000^{*}$ \\
\hline & Luis Carlos Galán & $1,73333^{*}$ \\
\hline & Gabriel García M. & $-2,04000^{*}$ \\
\hline & Ciudad Latina & $2,44667^{*}$ \\
\hline & Nuevo Compartir & $2,78667^{*}$ \\
\hline & Compartir & $2,83333^{*}$ \\
\hline San & La Despensa & $2,26667^{*}$ \\
\hline \multirow[t]{7}{*}{ Mateo } & León XIII & $2,11333^{*}$ \\
\hline & Buenos Aires & $2,82000^{*}$ \\
\hline & Ciudadela Sucre & $2,57667^{*}$ \\
\hline & Julio Cesar Turbay & $2,47000^{*}$ \\
\hline & Luis Carlos Galán & $2,70333^{*}$ \\
\hline & Gabriel García M. & $3,01000^{*}$ \\
\hline & Ricaurte & $1,80333^{*}$ \\
\hline Ricaurte & San Mateo & $1,80333^{*}$ \\
\hline \multirow[t]{4}{*}{ Las Villas } & Gabriel García M. & $1,75333^{*}$ \\
\hline & Ciudad Latina & $2,29000^{*}$ \\
\hline & Nuevo Compartir & $2,63000^{*}$ \\
\hline & Compartir & $2,67667^{*}$ \\
\hline Eugenio & La Despensa & $2,11000^{*}$ \\
\hline Díaz & León XIII & $1,95667^{*}$ \\
\hline \multirow[t]{5}{*}{ Castro } & Buenos Aires & $2,66333^{*}$ \\
\hline & Ciudadela Sucre & $2,42000^{*}$ \\
\hline & Julio Cesar Turbay & $2,31333^{*}$ \\
\hline & Luis Carlos Galán & $2,54667^{*}$ \\
\hline & Gabriel García M. & $2,85333^{*}$ \\
\hline
\end{tabular}

Se puede apreciar en la tabla 3, que la institución educativa Eugenio Díaz Castro fue la que presento mayor diferencia de medias del Índice Sintético de Calidad Educativa con las instituciones Ciudad Latina, Nuevo Compartir, Compartir, La Despensa, León XIII, Buenos Aires, Ciudadela Sucre, Julio Cesar Turbay, Luis Carlos Galán y Gabriel García Márquez.

En la figura 1, se observan las instituciones educativas oficiales y su ISCE para la Básica Primaria, las instituciones que presentaron mayor ISCE fueron: Santa Mateo, Cazucá, General Santander y León XIII. Las instituciones que presentaron menor ISCE para la Básica Primaria fueron: Buenos Aires, Nuevo Compartir y la Despensa. 


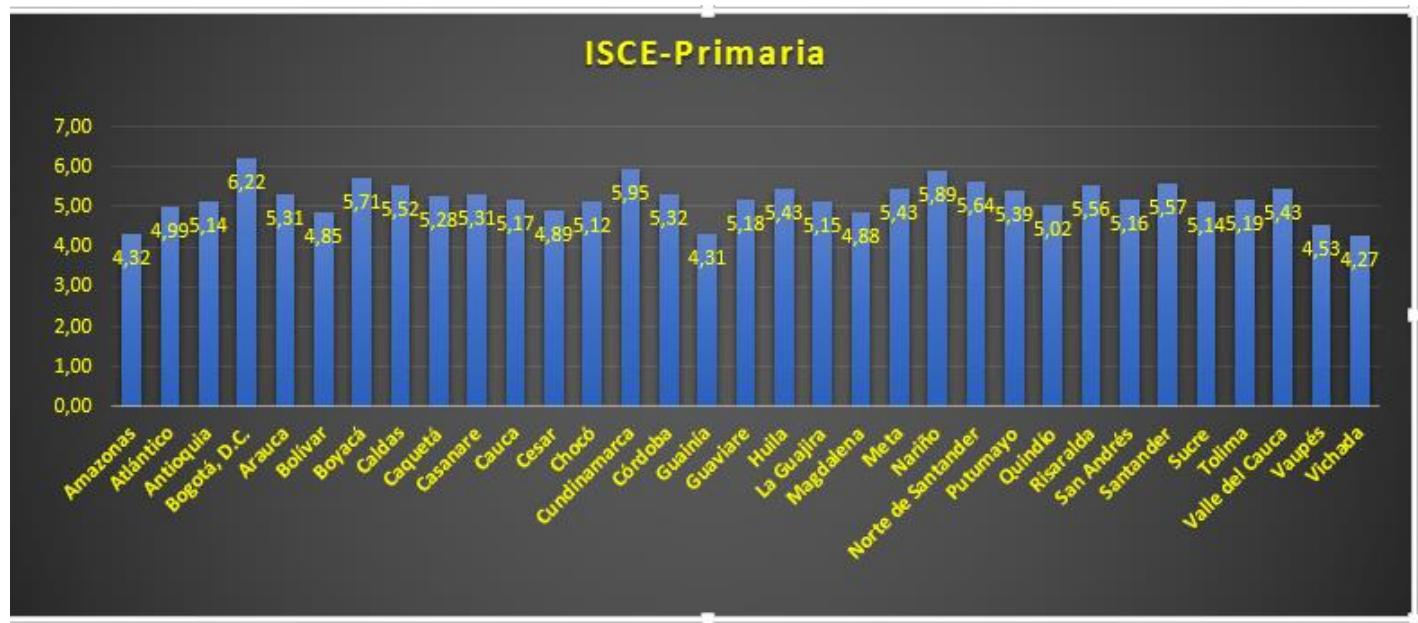

Figura 1. Índice Sintético de Calidad Educativa para Básica Primaria

Fuente: Autor

En la figura 2, se observan las instituciones educativas oficiales y su ISCE para la Básica Secundaria, las instituciones que presentaron mayor ISCE fueron: Manuela Beltrán, Integrado de Soacha y la Despensa. Las instituciones que presentaron menor ISCE para la Básica Primaria fueron: Gabriel García Márquez, Compartir y Ricaurte.

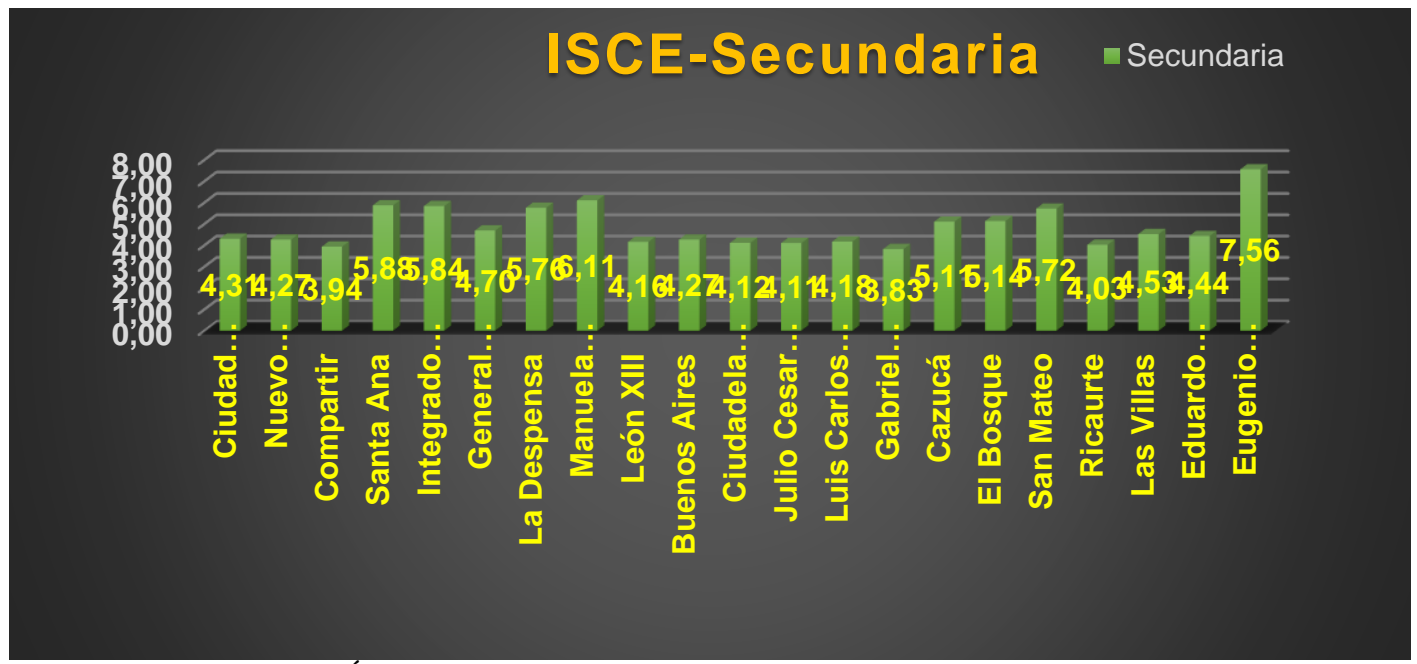

Figura 2. Índice Sintético de Calidad Educativa para Básica Secundaria

En la figura 3, se observan las instituciones educativas oficiales y su ISCE para la Básica Secundaria, las instituciones que presentaron mayor ISCE fueron: Eugenio Díaz Castro, Santa Ana y San Mateo. Las instituciones que presentaron menor ISCE para la Media fueron: Gabriel García Márquez, Luis Carlos Galán y Buenos Aires. 


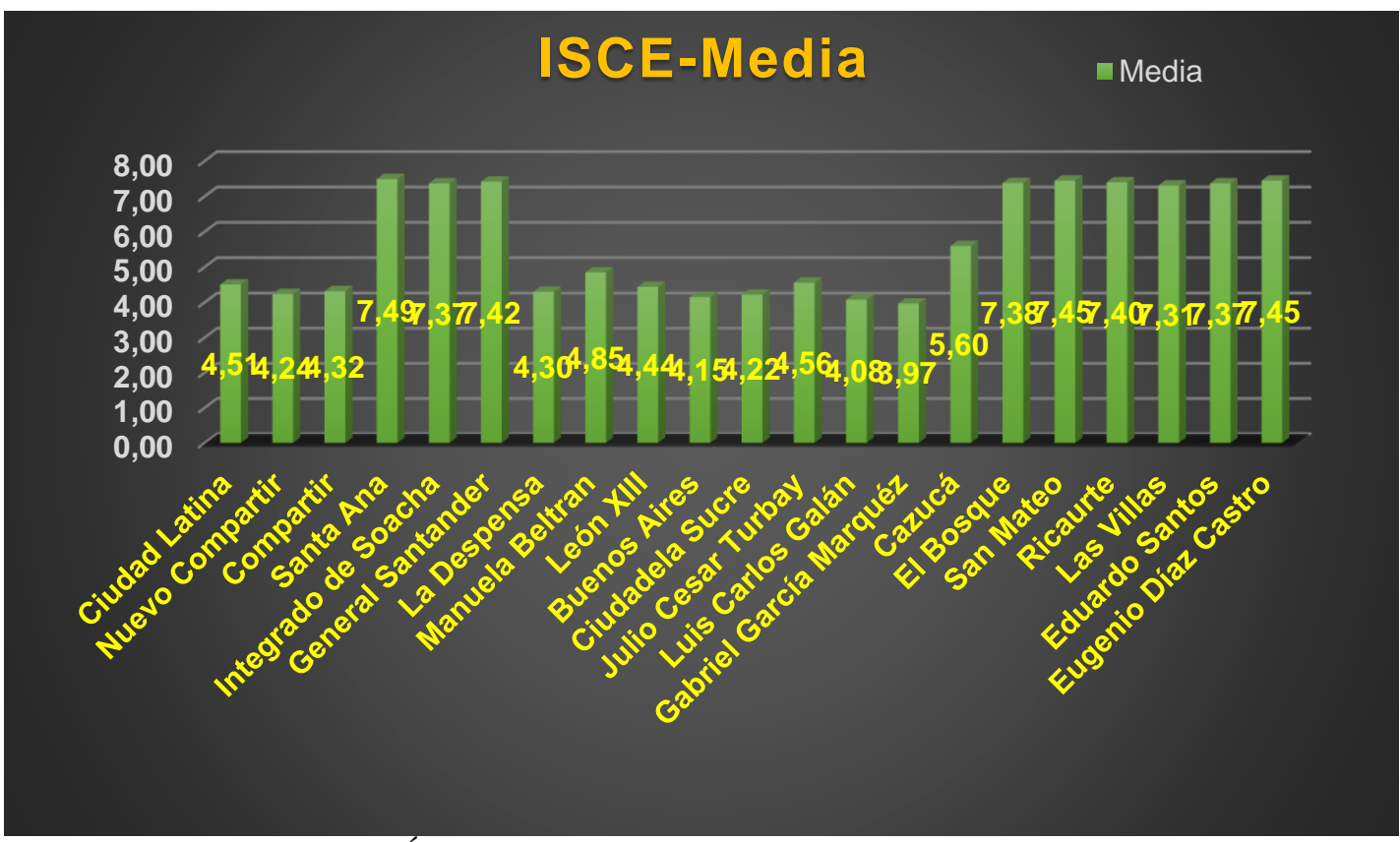

Figura 3. Índice Sintético de Calidad Educativa para la Media.

\section{Conclusión}

A partir del análisis estadístico de los datos aplicando la ANOVA, el p-valor de la prueba es igual a 0,12 es menor a $\alpha=0.05$, de esta manera se rechaza Ho, es decir que para al menos uno de los promedios es diferente de los demás del resultado obtenido por las instituciones educativas del municipio de Soacha-Cundinamarca en Índice Sintético de Calidad Educativa en el año 2017.

Las instituciones educativas con mejor promedio de Índice Sintético de Calidad Educativa para básica Primaria, básica secundaria y media fueron: Eugenio Díaz Castro con un promedio de 6,86, Santa Ana con 6,16 y General Santander con 6,09. Y las instituciones educativas con menor promedio fueron: Buenos Aires con 4,20 y Nuevo Compartir con 4,23 y Compartir con 4,19 .

Para tratar de mejorar el ISCE, se pueden mejorar las prácticas de aula en Matemáticas y Lenguaje, ejemplo de ello tenemos los trabajos realizados por (Díaz, 2016a; 2017a), con el uso de simuladores, logrando evidenciar una mejora en la enseñanza de las matemáticas, además se debe tener una formación permanente de los docentes en el conocimiento y apropiación de las tecnologías de la información y la comunicación. (Díaz, 2017b; 2017c).

Según (Universidad del Norte, 2015). Los ambientes de aprendizaje habrán de promover búsquedas críticas, donde la formación magistral sea reemplazada por opciones que conduzcan a una reflexión sobre la práctica, edificada desde la experiencia de los participantes; donde el lugar de alguien que da soluciones sea depuesto por una cimentación colectiva que reconoce los efectos diferentes que tienen las estrategias pedagógicas en cada grupo social.

Otra alternativa para mejorar el desempeño, el progreso, la eficiencia y el ambiente de aula son las salidas pedagógicas, de acuerdo con (Díaz, 2016b; 2016c), son una estrategia de 
enseñanza que crea en los estudiantes una visión general de los conceptos adquiridos en clase, además de propiciar el aprendizaje dinámico, ofrecer nuevos espacios para la enseñanza, la comprobación, el análisis y la construcción del conocimiento y permitir el contacto de los estudiantes con aptitudes científicas e investigativas.

\section{Bibliografía}

Díaz, J. (2016a). Aplicación PhET: estrategia de enseñanza-aprendizaje de fracciones equivalentes. Revista Criterios Vol.23, No1 (2016). En línea: http://www.umariana.edu.co/ojs-ditorial/index.php/criterios/article/view/1193

Díaz, J. (2016b). Planetario: Estrategia de aprendizaje. Revista Fedumar Pedagogía y Educación, 3(1), 117-125. (2016). En línea: http://www.umariana.edu.co/ojseditorial/index.php/fedumar

Díaz, J. (2016c). Clubes de Astronomía: Didáctica de enseñanza de la Ciencia y la Investigación. Revista Fedumar Pedagogía y Educación, 3(1), 127-137 (2016). En línea http://www.umariana.edu.co/ojs-editorial/index.php/fedumar

Díaz, J. (2017a). Importancia de la simulación Phet en la enseñanza y aprendizaje de fracciones equivalentes. Revista Educación y Desarrollo Social. Vol.11, No1. (2017). En línea: https://revistas.unimilitar.edu.co/index.php/reds/article/view/2011

Díaz, J. (2017b). Conocimiento y apropiación de las tecnologías de la información y la comunicación (TIC) en la formación docente. Revista Actualidades Pedagógicas. Número 69,121-131 (2017).En línea: https://revistas.lasalle.edu.co/index.php/ap/article/view/4066/3198

Díaz, J. (2017c). Simulador Applet Descartes: Como didáctica de enseñanza de la función cuadrática. . Revista Innova Research Journal. Vol. 2.Número 8, 69-78. En línea: http://www.journaluidegye.com/magazine/index.php/innova/article/view/256/342

Godoy, S. (2015). Índice Sintético de Calidad Educativa- ISCE. Recuperado el 20 de mayo de 2017 de http://www.ucc.edu.co/cali/prensa/2016/PublishingImages/Paginas/Jornada-deCalidad-Educativa-en-la-U

MEN (Ministerio de Educación Nacional) (2017). ¿Qué es el índice sintético de calidad educativa (ISCE)? Recuperado el 20 de mayo de 2017 de:http://www.colombiaaprende.edu.co/html/micrositios/1752/articles-349835_quees.pdf

Jaramillo, J. (2017). Siempre día-e.Recuperado el 20 de mayo de 2017 de: http://aprende.colombiaaprende.edu.co/siemprediae 
El Tiempo. (2017). Colombia superó las metas en educación para 2017. Recuperado el 20 de mayo de 2017 de: http://www.eltiempo.com/vida/educacion/resultados-del-indice-sintetico-decalidad-educativa-2017-86552

Universidad del Norte. (2015). Propuesta de Evaluación con Carácter Diagnóstico Formativa ECDF. Recuperado el 22 de junio de 2017 de: http://aprende.colombiaaprende.edu.co/sites/all/modules/mapa/docs/curso_ECDF/UNIN ORTE_curso\%20ECDF.pdf

Zambrano, M (2015). Alcances e inconsistencias del Índice Sintético de Calidad Educativa (ISCE), diseñado y aplicado por el Ministerio de Educación de Colombia en el año 2015. $\begin{array}{llllll}\text { Recuperado el } & 20 \text { de } & 2017 & \text { de: }\end{array}$ http://acofade.org/documentos/notiacofade/Indice\%20sinte\%C3\%ACtico\%20de\%20calid ad\%20educativa_MEN_Colombia.pdf 